\title{
MICROMEGAS calibration for ACTAR TPC
}

\author{
B. Mauss ${ }^{1}$, a , T. Roger ${ }^{1}$, J. Pancin ${ }^{1}$, S. Damoy ${ }^{1}$, and G. F. Grinyer ${ }^{1}$ \\ ${ }^{1}$ Grand Accélérateur National d'lons Lourds (GANIL), CEA/DSM-CNRS/IN2P3, Bvd Henri Becquerel, 14076 Caen, France
}

\begin{abstract}
Active targets, such as the ACtive TARget and Time Projection Chamber (ACTAR TPC) being developed at GANIL, are detection systems that operate on the basis of a time projection chamber but where the filling gas also serves as a thick target for nuclear reactions. In nuclear physics experiments, the energy resolution is of primary importance to identify the reaction products and to precisely reconstruct level schemes of nuclei. These measurements are based on the energy deposited on a pixelated pad plane. A MICROMEGAS detector is used in ACTAR TPC for the ionization electron collection and amplification, and it is a major contributor to the energy dispersion through, for example, inhomogeneities of the amplification gap. A variation of one percent in the gap can lead to an amplitude variation of more than two percent which is of the same order as the resolution obtained with an energy deposition of $5 \mathrm{MeV}$. One way to calibrate the pad plane is through the use of a two dimensional source scanning table. It is used to calibrate the gain inhomogeneities and, using MAGBOLTZ calculations, deduce the corresponding gap variations. The inverse of this method would allow the relative gain variations to be calculated for the different gas mixtures and pressures used in experiments with ACTAR TPC.
\end{abstract}

\section{Introduction}

Nuclear matter under extreme conditions of excitation energy or extreme isospin exhibits properties which strongly differ from those of nuclei near stability. The appearance of nuclear matter haloes near the drip lines or the formation of clusters at high excitation energies are examples of such exotic phenomena. Access to these extreme conditions requires the study of nuclei far from stability that are produced with low beam intensities or through reactions with small cross sections. The use of very efficient detection systems and thick targets is needed to cope with these difficulties. In an active target, detection of the reaction products is performed inside the target, the detection efficiency and effective target thickness are increased without losing resolution since the position of the reaction inside the target is measured.

Active targets and Time Projection Chambers (TPC) were introduced in nuclear physics about 20 years ago with the detector IKAR $[1,2]$ in GSI. It was followed by the MAYA active target at GANIL [3-5] and the CENBG TPC $[6,7]$ used for decay studies. In order to gain in sensitivity and precision on the reconstruction of trajectories, the next generation active target ACTAR TPC is now being developed at GANIL. ACTAR TPC is foreseen to be used for direct and resonant reactions, and decay studies. A demonstrator of this future active target has been built. Its working principle is the following: ionization electrons produced along charged particle tracks drift under the influence of an electric field to a readout plane composed of 2048 pads of $2 \times 2 \mathrm{~mm}^{2}$ each. The drift field

\footnotetext{
a e-mail: mauss@ganil.fr
}

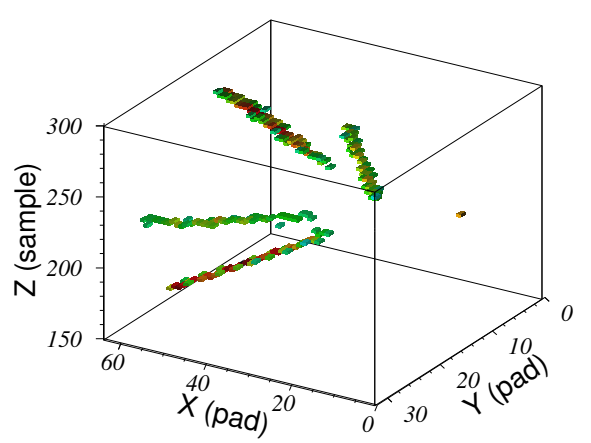

Figure 1. Example of a ${ }^{12} \mathrm{C}\left({ }^{4} \mathrm{He},{ }^{4} \mathrm{He}\right){ }^{12} \mathrm{C}^{*} \rightarrow 3 \alpha$ event recorded with the ACTAR TPC demonstrator. The color scale corresponds to the value of the charge deposited per pad. The $6.6 \mathrm{MeV} / \mathrm{u}{ }^{12} \mathrm{C}$ beam entered the active volume from the right, along the $\mathrm{X}$ axis. The chamber was filled with a $\mathrm{He}: \mathrm{iC}_{4} \mathrm{H}_{10}$ 90:10 gas mixture with a pressure of 1 bar.

cage is $17 \mathrm{~cm}$ high with 168 evenly-spaced metallic wires on the four sides. By degrading the wires potential from the cathode potential to the MICROMEGAS potential, this field cage permits to have an homogeneous vertical electric field. The amplification of the primary ionization electrons is achieved through the use of a $220 \mu \mathrm{m}$ amplification gap obtained with a bulked MICROMEGAS [8, 9], which matches the requirements of the TPC in terms of timing, gain, types of gases used and operating pressure [10]. The readout of the pads is performed using GET digital elec- 


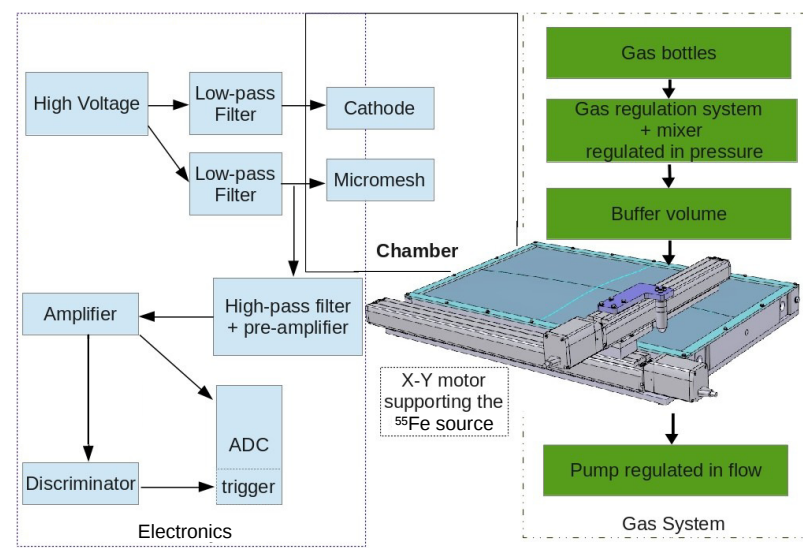

Figure 2. Test bench layout, with the electronics for the supply of the cathode and mesh (upper part) and the reading of the signal on the mesh (lower part). In green the pumping system for the regulation in pressure of the gas inside the chamber.

tronics [11], thus enabling a full 3D reconstruction of the tracks, as illustrated in Figure 1.

The identification of the reaction products can be achieved using the correlation between their average energy loss measured along their path and their total energy. The latter can be estimated either by reconstructing the range of the particles if they stop in the gas or using auxiliary detectors (such as silicon detectors) if the particles escape the active volume. The identification capabilities of the active target therefore depend on the local gain variations which arise from inhomogeneities of the gap over the whole pad plane.

In this article, we propose a method to correct gain inhomogeneities for each gas/target and pressure. This method is based on the measurement of the response of the MICROMEGAS to an ${ }^{55} \mathrm{Fe} \mathrm{X}$-ray source as a function of the position of the source over the pad plane. This will be described in Section 2. The reconstruction of the relative gap for each pad of the pad plane using MAGBOLTZ calculations to obtain the associated gain corrections for any gas/target is developed in Section 3.

\section{Experimental set-up}

The MICROMEGAS was installed in a chamber where the gas pressure was regulated to be stable at better than \pm 0.1 mbar. A drift gap of $2 \mathrm{~cm}$ was achieved through the placement inside the chamber of a meshed cathode above the MICROMEGAS. An X-Y motorized holder was used to position the collimated ${ }^{55} \mathrm{Fe} \mathrm{X}$-ray source $7 \mathrm{~cm}$ above each pad with a precision of $\pm 0.1 \mathrm{~mm}$, the X-ray beam irradiating a zone of $3 \times 3$ pads. The irradiated zone has the shape of a 2D Gaussian function centered on the central pad, which collects more than $60 \%$ of the events. A similar method was used to calibrate the gain of the MICROMEGAS detectors for the T2K experiment [12]. The recorded events correspond to the photoionization of the gas. The photoelectrons drift towards the pad plane and are amplified in the MICROMEGAS gap, between the mi-

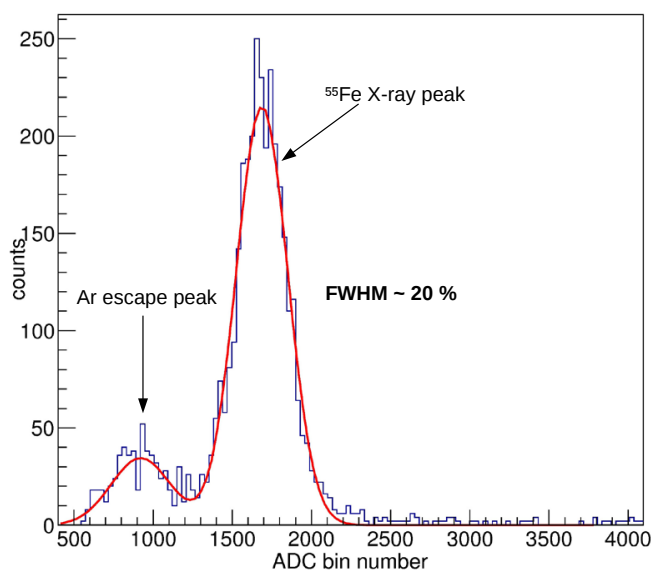

Figure 3. Typical ${ }^{55} \mathrm{Fe} \mathrm{X}$-ray source spectrum in $\mathrm{Ar}: \mathrm{iC}_{4} \mathrm{H}_{10}$ $97.15: 2.85$ at $P=1021 \mathrm{mbar}$ and $V_{\text {mesh }}=-420 \mathrm{~V}$.

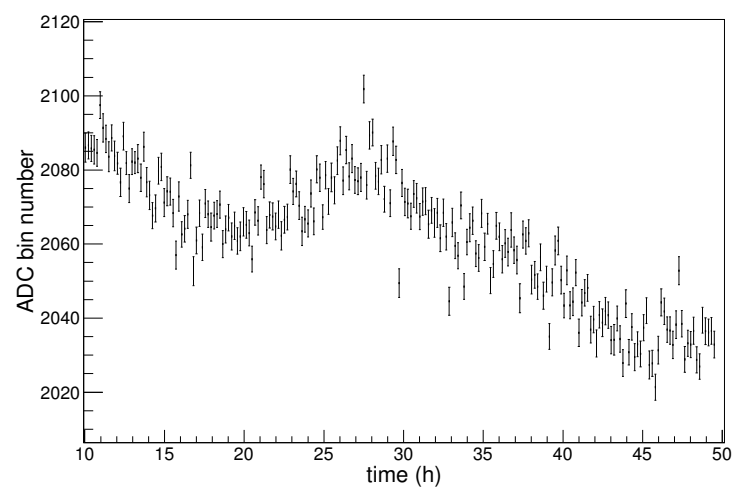

Figure 4. Variations of the gain at a reference position over a two-day period. The variation in gain is $\simeq 2.5 \%$.

cromesh and the pad plane. The avalanche signal from the micromesh is sent to a pre-amplifier and an amplifier. If the signal is large enough, a discriminator sends a trigger to the ADC that records the charge. The blue part of Figure 2 shows a schematic of the acquisition chain.

During the calibration, 1500 events were acquired for each pad. A typical spectrum from a single measurement, which takes approximately $90 \mathrm{~s}$, is shown in Figure 3. The centroid position of the $5.9 \mathrm{keV}$ photoionization peak was obtained through a double Gaussian fit to this peak and the Argon escape peak observed on the figure. The centroid position was associated to the corresponding $(\mathrm{x}, \mathrm{y})$ position of the pad.

Over a few hours of data taking, the gas characteristics may change slightly because of temperature variations. To take these effects into account, a systematic measurement every 30 minutes was performed on a reference position in order to realign the amplitude response. An example of such variations are shown in Figure 4 where large day/night variations were observed. The gain variation of $(2.5 \pm 0.8) \%$ is coherent with a temperature variation on the order of $1 \mathrm{~K}$ but a more precise evaluation is being investigated.

The MICROMEGAS was bulked [9] using 4 layers of 


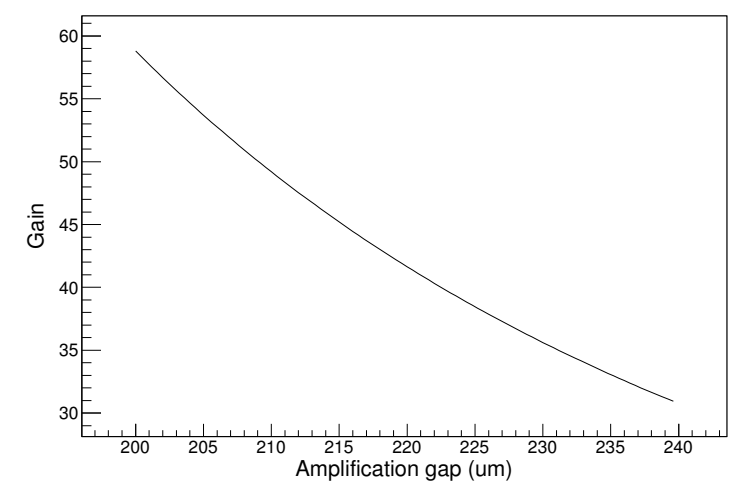

Figure 5. Gain as a function of the MICROMEGAS amplification gap as obtained from MAGBOLTZ calculations for an Ar: $\mathrm{iC}_{4} \mathrm{H}_{10} 97.15: 2.85$ mixture at $P=1020 \mathrm{mbar}$ and $T=24^{\circ} \mathrm{C}$

vacrel below the mesh (provided by the CERN PCB Workshop). A pillar of $300 \mu \mathrm{m}$ diameter is placed at the center of each pad, which should not have any effect on the measurements. This unusual gap size of over $200 \mu \mathrm{m}$ was chosen because of the wider range of operational pressure [10]. For the calibration, the detector was operated at a voltage $V_{\text {mesh }}=-420 \mathrm{~V}$ with a constant drift field of about $300 \mathrm{~V} \cdot \mathrm{cm}^{-1}$. The chamber contained a mixture of Ar: $\mathrm{iC}_{4} \mathrm{H}_{10}$ 97.15:2.85 supplied by a premixed bottle at a few mbars above atmospheric pressure. In this context, the absolute gain of the MICROMEGAS was found to be of the order of $8 \cdot 10^{2}$. Measurements at each position had a resolution of about $20 \%$ FWHM.

\section{Calibration of the MICROMEGAS}

\subsection{Analysis and results}

From the centroid position obtained for each pad position, it was then possible to obtain a relative correction in gain of the pad plane. It was referred in Section 1 that, depending on the target required, the gas inside the chamber will change. The software MAGBOLTZ [13] can be used to calculate the gas characteristics such as the electron drift velocity or the Townsend coefficient as a function of the electric field and for most of the gas mixtures used in detection, at fixed temperature and pressure [14]. Thus, it is possible to adapt the correction factor to the particular target/gas used inside the chamber.

The Townsend coefficient $\alpha$ was calculated for several values of the electric field and a cubic spline was used for the interpolation. Taking the working voltage $V_{\text {mesh }}$ of the detector, the Townsend coefficient was evaluated as a function of the amplification gap $d$. The gain $G$ was then calculated from the approximation $G=e^{\alpha d}$. The size of the amplification region $d$ changes the value of the amplification through the active length and through the Townsend coefficient that depends on the electric field. In figure 5, the gain is plotted as a function of the gap in our region of interest, around $220 \mu \mathrm{m}$. This gap value was obtained through optical measurements of the gap size performed previously. Using a similar curve for any gas and pressure

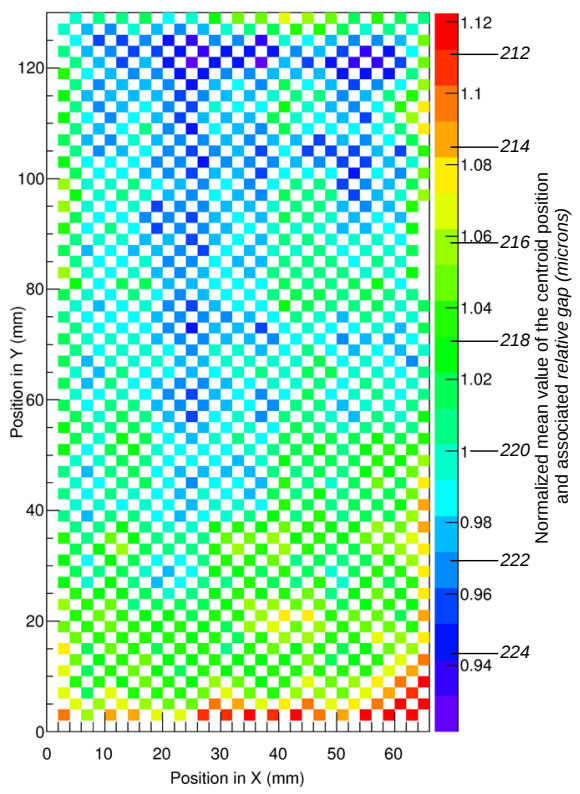

Figure 6. Result obtained for the $\mathrm{Ar}_{\mathrm{iC}} \mathrm{H}_{4} \mathrm{H}_{10}$ 97.15:2.85 gas mixture at 1021 mbar. The gap, as calculated from MAGBOLTZ using $220 \mu \mathrm{m}$ as reference, is also indicated on the color scale.

used, it is possible to convert from gap to gain and vice versa.

Some effects like the Penning effect are not completely taken into account for certain gas mixtures in MAGBOLTZ calculations. These effects are assumed to be constant within the range of temperature/gap considered in this study. Therefore, the average gain that was deduced was then scaled to the one calculated by MAGBOLTZ for a gap of $220 \mu \mathrm{m}$. Gap variations were then obtained using the curve of Figure 5. It confirms that the gain calculated by MAGBOLTZ is very different from the measured gain. This discrepancy can easily be explained by variations of the Townsend coefficient smaller than a factor of two and due for example to the Penning effect [15]. The result of a scan for every other pad is shown in Figure 6 with the relative normalized gain and the corresponding gaps ranging from 212 to $224 \mu \mathrm{m}$. The uncertainties for the gap values were extracted from the uncertainty on the measured amplitude response. The uncertainty on the centroid determination was about $0.3 \%$ and propagation to the uncertainty on the gap led to uncertainties below $0.4 \mu \mathrm{m}$. To study the sensitivity of the method on the chosen mean gap, the calculation was also performed using mean gaps of 200 and $240 \mu \mathrm{m}$. The subsequent gap variations were only lowered and increased by $1 \mu \mathrm{m}$, respectively.

\subsection{Application to other gases and mixtures}

Once a map of the amplification gap over the pad plane is obtained with this calibration, it is possible to calculate gain variations for other gases. Once again, there are unaccounted for effects in MAGBOLTZ and it is only possible to calculate relative gain variations. However, considering that the contributions of these effects is constant with small gap variations, they don't influence the relative variations 
of the gain. This will be verified using another mixture like $\mathrm{Ne}+\mathrm{iC}_{4} \mathrm{H}_{10}$. The new relative gain variations calculated using MAGBOLTZ should be the same as the new relative gain variations measured in this mixture.

\section{Conclusion and outlooks}

The technique presented here can be used on any MPGD detector to efficiently verify the homogeneity of the amplification stage. Relative corrections can be performed for any gas/target described by MAGBOLTZ which can be useful for any kind of active target or TPC.

The corrections calculated with MAGBOLTZ will be compared with the mapping of the same pad plane in a different gas mixture to validate the method. If successful, the gap/gain corrections will be used in the data analysis of the experiment performed with the ACTAR TPC demonstrator to realign the pad plane response and improve the energy resolution. The scanning table is adapted to the final ACTAR TPC pad planes of 16384 channels with sizes $25.6 \times 25.6 \mathrm{~cm}^{2}$ and $12.5 \times 51.2 \mathrm{~cm}^{2}$. Acceptance tests will be performed using this scanning table and the gaps will be calculated to be able to correct the local gains.

\section{Acknowledgments}

The research leading to these results has received funding from the European Research Council under the Eu- ropean Union's Seventh Framework Program (FP7/20072013)/ERC grant agreement $n^{\circ} 335593$.

\section{References}

[1] A.V. Dobrovolsky et al., Nucl. Phys. B 214, 1 (1983)

[2] S. Ilieva et al., Nucl. Phys. A 875, 8 (2012)

[3] C.-E. Demonchy et al., Nucl. Instr. and Meth. A 431, 341 (2007)

[4] T. Roger et al., Phys. Rev C 79, 031603(R) (2009)

[5] M. Vandebrouck et al., Phys. Rev. Lett. 113, 032504 (2014)

[6] B. Blank et al., Nucl. Instr. and Meth. A 613, 65 (2010)

[7] P. Ascher et al., Phys. Rev. Lett. 107, 102502 (2011)

[8] I. Giomataris et al., Nucl. Instr. and Meth. A 376, 29 (1996)

[9] I. Giomataris et al., Nucl. Instr. and Meth. A 560, 405 (2006)

[10] J. Pancin et al., Nucl. Instr. and Meth. A 735, 532 (2014)

[11] E.C. Pollaco et al., Physics Procedia 37, 1799 (2012)

[12] A. Delbart, Nucl. Instr. and Meth. A 623, 105 (2010)

[13] magboltz.web.cern.ch/magboltz/

[14] garfield.web.cern.ch/garfield/help/garfield_40.html

[15] M.A. Chefdeville, PhD thesis, Univ. of Twente, http://doc.utwente.nl/62815/ (2009) 